\title{
Globe
}

Revue internationale d'études québécoises

\section{Jean-Marc Piotte et Jean-Pierre Couture, Les nouveaux visages \\ du nationalisme conservateur au Québec, Montréal, Québec-Amérique, 2012}

\section{Cédric Nadon}

Volume 16, numéro 2, 2013

URI : https://id.erudit.org/iderudit/1025222ar

DOI : https://doi.org/10.7202/1025222ar

Aller au sommaire du numéro

Éditeur(s)

Globe, Revue internationale d'études québécoises

ISSN

1481-5869 (imprimé)

1923-8231 (numérique)

Découvrir la revue

Citer ce compte rendu

Nadon, C. (2013). Compte rendu de [Jean-Marc Piotte et Jean-Pierre Couture, Les nouveaux visages du nationalisme conservateur au Québec, Montréal, Québec-Amérique, 2012]. Globe, 16(2), 172-174.

https://doi.org/10.7202/1025222ar d'utilisation que vous pouvez consulter en ligne.

https://apropos.erudit.org/fr/usagers/politique-dutilisation/ 


\section{Jean-Marc Piotte et Jean-Pierre Couture \\ Les nouveaux visages du nationalisme conservateur \\ au Québec, Montréal, Québec-Amérique, 2012.}

Dans Les nouveaux visages du nationalisme conservateur au Québec, Jean-Marc Piotte et Jean-Pierre Couture tentent de cerner la montée, au cours des dernières années au Québec, d'une nouvelle forme de nationalisme conservateur. Celle-ci aurait émergé à la suite de différents événements, tels l'ascension, en 2007, de l'ADQ (Action démocratique du Québec) au rang de parti d'opposition officielle, la crise des accommodements raisonnables et le dépôt, l'année suivante, du rapport Bouchard-Taylor. Ce courant aurait pris la place dominante qu'occupait auparavant, depuis la défaite référendaire de 1995, le nationalisme pluraliste. Le but du présent ouvrage est double: identifier, d'abord, les plus importantes figures de ce courant et offrir une critique des éléments conservateurs de leurs thèses; démontrer, ensuite, l'existence d'un réseau de diffusion de ces thèses, qui s'organise autour de différents organes médiatiques.

Pour ce faire, les auteurs ont ciblé six intellectuels qu'ils jugent comme étant les plus importants représentants de ce néoconservatisme : Jacques Beauchemin, Joseph-Yvon Thériault, Éric Bédard, Marc Chevrier, Gilles Labelle et Stéphane Kelly. Un chapitre est consacré à chacun de ces penseurs; on y analyse leurs écrits et leurs déclarations dans le but d'identifier et de critiquer les thèses qui participent à cette mouvance néoconservatrice. Bien que Piotte et Couture admettent, en début de livre, que ces intellectuels possèdent des positions bien différentes, ils affirment que ces derniers partagent tous «des dénominateurs communs» qui "permettent de les regrouper sous le chapeau du nationalisme néoconservateur : le passéisme, la critique conservatrice de la modernité, l'épistémologie idéaliste, l'oubli ou le rejet de l'apport des sciences sociales et l'euphémisation de leur conservatisme " (p. 12). À travers cette entreprise, les auteurs ont donc pour objectif de démontrer les liens entre ces dénominateurs communs et les thèses étudiées dans le livre.

Malgré la pertinence d'un tel exercice, les analyses présentées ne sont pas toujours soutenues par une démonstration argumentative très convaincante. On sent, par moments, que les auteurs présument que le lecteur est a priori en accord avec les thèses énoncées par l'ouvrage et qu'il est donc inutile d'élaborer davantage. Cela se traduit en un certain nombre de 
généralisations que l'on retrouve tout au long de l'ouvrage. Ne donnons que quelques exemples. Lors d'un passage traitant de la critique que fait Marc Chevrier du système d'éducation découlant de la commission Parent, les auteurs expriment leur désaccord sur la question de l'éducation des élèves, affirmant que " [l]a psychologie nous a enseigné les processus d'apprentissage de chacun" (p. 96). Piotte et Couture ne mentionnent toutefois jamais de quels enseignements en psychologie il s'agit. Plus tard, lors d'un passage traitant des positions de Gilles Labelle sur la Révolution tranquille, ils mentionnent que celles-ci sont formulées "[c] ontre les analyses sociopolitiques" (p. 111). On ne trouve toujours aucune mention de quelles analyses sociopolitiques il s'agit. Lors d'une discussion sur l'idée que Beauchemin a de la place du pouvoir au sein de la société, les auteurs mentionnent que : «Foucault affirmait que le pouvoir était partout et nulle part. Mais le peuple est-il foucaldien? Les études empiriques ne démontrent-elles pas que le pouvoir déterminant dans la société est toujours exercé par le Capital et l'État?» Les auteurs ouvrent ainsi une discussion sur la place du pouvoir dans la société pour la refermer aussitôt. Encore une fois, ils ne mentionnent jamais de quelles études empiriques il s'agit et affirment cela comme s'il y avait consensus, en sciences sociales, sur la question du pouvoir. L'ouvrage contient plusieurs exagérations, généralisations et raccourcis théoriques, ce qui nuit considérablement à la démonstration. Précisons que, malgré cette faille importante au plan de l'argumentation, les auteurs réussissent tout de même à noter certains problèmes, au sein des thèses de ces six intellectuels, qui mériteraient un examen sérieux. Il est donc dommage que ces critiques ne soient pas accompagnées d'une argumentation plus serrée.

La dernière partie du livre est consacrée à la démonstration de l'existence d'un "réseau intellectuel hégémonique» (p. 145) qui servirait de plateforme à l'expression, dans le domaine public, de ce néoconservatisme. Les auteurs sont bien conscients de la parure conspirationniste d'une telle affirmation et se défendent, de prime abord, de tomber dans la théorie du complot. Ils préfèrent utiliser les concepts de «sociabilité intellectuelle et littéraire» (p. 149) et d'" auteur collectif» (p. 150).

L'usage du concept d'auteur collectif, jumelé à l'insistance, tout au long du livre, sur ce qui rapproche les auteurs plutôt que sur ce qui les différencie, laisse transparaitre une image trop homogène des penseurs, malgré l'admission, mentionnée rapidement en début de livre, que leurs positions diffèrent sur plusieurs points. S'il s'agit d'un "groupe très structuré » (p. 16) hégémonique, cela reste à démontrer. Il serait toutefois certainement difficile de nier l'existence d'une tendance néoconservatrice, d'un courant de la 
"nouvelle sensibilité historique ", et de sa présence importante dans les discours publics et académiques québécois. Il est aussi vrai que plusieurs de ces auteurs, sur certaines questions, vont collaborer et se porter à la défense des idées qu'ils partagent, comme cela a été le cas, par exemple, lors des débats entourant la publication du rapport Bédard-D’Arcy. Sur ce point, le livre s'avère plus convainquant.

Malgré ses lacunes, l'ouvrage n'est pas sans mérite. Le livre est bien documenté, contenant une bibliographie exhaustive constituée, en grande majorité, de nombreuses publications des intellectuels critiqués. La publication de ce titre est d'ailleurs pertinente, considérant la conjoncture actuelle, puisque les thèmes abordés sont au cœur des débats en cours au Québec. En témoigne, par exemple, l'ampleur de la polémique entourant la charte des valeurs du Parti québécois : y sont abordées les questions du pluralisme, de l'identité, de la culture, de la nation, de l'histoire, de la mémoire et du rapport au passé. Enfin, Les nouveaux visages du nationalisme conservateur au Québec arrive à démontrer l'existence bien réelle d'un courant néoconservateur au Québec et à cerner un nombre de problèmes au sein des thèses qui animent ce mouvement. Il échoue toutefois à exposer ces thèses avec précision et à y répondre d'un argumentaire convainquant et appuyé.

Cédric Nadon Université du Québec à Montréal 